\title{
ENCITE: A model to help Voluntary Organisations use ICT effectively
}

TJ McDonald

Waterford Institute of Technology

Corresponding Author.

tmcdonald@wit.ie

\begin{abstract}
Local Voluntary Organisations (LVOs) play an important role in Irish society. LVOs can be based on community, political, sporting, recreational or civil needs. These organisations facilitate information dispersion, social interaction, employment creation and service provision in communities. However, many of these LVOs are struggling to exist and maintain their role in Irish society; one of the primary reasons for this problem is their reluctance to embrace and use Information and Communication Technology (ICT) effectively. Voluntary organisations need help to identify a role for ICT and to subsequently design and develop technical solutions. The ENCITE model will facilitate Irish LVOs to plan and design applications that will support their operation and empower their members.
\end{abstract}

\section{Introduction}

Local Voluntary Organisations (LVOs) are an important part of Irish peoples' lives and make a valuable contribution to society. However, many Irish LVOs are struggling to exist and maintain their relevance to their communities. In particular, these organisations recognise that they have been slow to adopt and use Information and Communication Technology (ICT) and this inaction has a negative impact on their operation and development, costing them time and money and resulting in numerous inefficiencies, all of which has diminished their contribution to their communities. A typical example of the slow adoption of technology offered by some organisations is the paucity of email usage among its members, both internally and externally. This paper proposes the ENCITE model to help LVOs adopt and use ICT effectively. The ENCITE model is based on data gathered from a qualitative study conducted with over 60 informants. The ENCITE model will support LVOs to plan, design

McDonald, T.J. (2016). ENCITE: a model to help voluntary organisations use ICT effectively. The Journal of Community Informatics, 12(1), 142-151.

Date submitted: 2015-09-16. Date accepted: 2015-11-19.

Copyright (C), 2016 (the authors as stated). Licensed under the Creative Commons AttributionNonCommercial-ShareAlike 2.5. Available at: www.ci-journal.net/index.php/ciej/article/view/1211 
and develop technical solutions that solve their problems in an inclusive and iterative manner; it will facilitate voluntary organisations to develop a technical plan and help their members

understand the potential of ICT and perhaps more importantly define a role for it in their organisation. The ENCITE model will help voluntary organisations to include their members' views, needs and expectations in the design of technical applications and subsequently in the creation of a plan to create in-house or outsourced ICT solutions.

\section{Local Voluntary Organisations in Ireland}

In Ireland, the term LVO is predominantly used to describe locally organised voluntary effort. Irish LVOs include parish committees, Gaelic Athletic Association (GAA) clubs, soccer and athletics organisations, parents' associations, The Irish Farmers Association (IFA), Irish Country Women's Association (ICA) and a plethora of other groups, some unique to a geographical area1. In general, Irish LVOs tend to have county, provincial and national levels that support their operation and often dictate their function. For example, the local GAA club is affiliated to the county GAA board that in turn is a member of the national GAA executive. LVOs consist of members who pay a membership subscription each year. Some of these members may serve as officers on the management committee elected at an annual general meeting as decreed in the organisation's constitution. Voluntary organisations are an integral part of the social fabric in Ireland and play an important role in integrating people into communities (O'Donnell, 1999). However, for the vast majority of Irish LVOs a number of problems are stymieing their development and growth. These problems include: diminishing membership, declining relevance to community, decreasing levels of altruism among members, new government legislation (such as drink driving restrictions and smoking bans), social inclusion and cultural problems, accessing external resources and supports and increasing financial constraints. These problems are exacerbated by technical troubles such as a lack of ICT infrastructure, accessing technical expertise, and confusion over emerging software trends (O’Donnell, 2000; Gilligan, 2005; Dochas, 2006; Mc Donald, 2012). In addition there is a paucity of opportunities for LVO members to upskill, reskill or improve their education in an environment conducive to learning (IRL, 2010). Finally, there is a lack of co-operation and collaboration between LVOs, resulting in each unnecessarily operating independently and often replicating the mistakes of others, to their own detriment (IRL, 2010; Mc Donald, 2012).

McDonald (2012) argues that the deployment of appropriate forms of ICT can help Irish LVOs address many of their problems. However, McDonald suggests that before these organisational problems can be tackled with various forms of technology, their members need to become more technically competent. The potential of ICT is not fully understood by many members of these voluntary organisations, which makes their adoption of technology difficult, if not impossible. Consequently, Irish voluntary organisations are slow to adopt technology or even worse select unsuitable forms of ICT, a decision that negatively impacts their future use of it (Gilligan, 2005; CUITA, 2010). The Wheel (2010) caution that the

1 Certain areas of Ireland would have LVOs that are unique to that community, e.g. Irish language groups in parts of Cork, Galway and Kerry. 
adoption and diffusion of technology into Irish voluntary organisations needs to be carefully planned, with particular emphasis on recognising and embracing the impact that organisational culture has on the success of the initiative.

The Irish government also accepts the importance of addressing community needs through the development of voluntary organisations. Former Taoiseach (Irish Prime Minister) Mr. Bertie Ahern (2000) stated that ICT will play an important role in helping Irish LVOs address their concerns and facilitate their strategic development. A number of national initiatives were developed at the beginning of the millennium to enable and support communities such as: "A Framework for Supporting Voluntary Activity' (2000), which facilitated the creation of a number of support agencies; 'The Access, Skills and Content' (ASC) initiative (2001), which focused on the development of local communities. Other initiatives like 'e-Inclusion' (2005) were created by the European Union (EU). This initiative placed the spotlight on Internet provision and reduction of the digital divide, particularly in rural areas. These initiatives have concluded, with no credible replacements owing to the dire financial situation in Ireland and across Europe in recent times.

\section{Data Gathering and Analysis}

To gather data to aid the development of the ENCITE model, knowledgeable informants were selected in equal proportion from the three largest sectors of the Irish voluntary landscape: community, sporting, and rural and agricultural. Forty seven informants were interviewed for on average one hour in duration and a further fourteen participated in two focus groups, which took over two hours to complete respectively. All informants bar six (these were paid officers) volunteered at different 'levels' 2 in the community and voluntary sector; some were secretaries, several were treasurers, whilst others were committee members. This variety in informant ensured a broader understanding of how each 'level' viewed and used ICT for exclusive and mutual benefits. Some of the informants were members with an ICT background, whilst others were those who recognised the important potential role that ICT could play within their LVO and were motivated to embrace it. The initial cohort of informants was selected on the basis of the researcher knowing them from his previous work in the voluntary sector, a technique advocated by Babbie and Mouton (2001). Subsequently, the selection of informants largely followed a snowballing approach, where informants were selected primarily on the suggestion of others, a purposive sampling technique proposed by Polkinghorne (2005). The data gathered from the informants primarily focused on their views, uses, experiences and expectations of ICT currently and moving into the future. This data was analysed using hermeneutics as the primary analysis technique. Cognitive maps were used to graphically illustrate the findings. Subsequently, NVivo was used to complement the analysis and to identify any findings that may have been previously missed. Many findings emerged from the rich dataset. These findings provided an in-depth understanding of the concepts and activities that informants and participants felt facilitated the adoption and use of ICT services by Irish LVOs and their members. The concepts and activities were used to create the ENCITE model.

\footnotetext{
${ }^{2}$ The term 'levels' indicates the various areas of Irish LVOs, specifically local, county, regional and national.
} 


\section{Potential concepts and activities for inclusion in the model}

This section identifies the concepts and activities specified by informants for inclusion in the proposed model. Each concept and activity contributes in some way to the adoption and diffusion of ICT in Irish LVOs. The contribution of each concept and activity needs to be understood, before combining them together to develop a coherent and useful model.

\section{a. Strengths, Weaknesses, Opportunities and Threats (SWOT) analysis}

Many informants subscribed to the validity of using a SWOT analysis, although it is a relatively dated technique primarily used for strategic planning, several Community Informatics (CI) practitioners suggest that the strengths of a LVO need to be mobilised in order to allow them to develop as an organisation and achieve their objectives (Kretzmann \& Mc Knight, 1993; Anderson, 2000 \& Morse, 2004). A SWOT analysis identifies the strengths, which are the assets that the organisation may use to achieve its objectives; weaknesses, which identify the internal factors that obstruct organisations achieving their objectives; opportunities, which are the factors that may help the organisation achieve its objectives; and threats, which are the external conditions that are harmful to an organisation achieving its aims.

\section{b. Application Development Plan}

Informants comment that a plan is needed to guide the integration of ICT into LVOs. Primarily, the plan needs to be based on the findings of the SWOT analysis, which should identify a prioritised set of technological objectives that will support and improve the operation of the LVO. Several notable CI researchers espoused the importance of developing a plan for the diffusion of ICT into LVOs (O'Donnell, 2001; Carroll \& Rosson, 2007; Toyama, 2009). In particular, Carroll and Rosson (1996; 2007) demonstrated through the Blacksburg Electronic Village (BEV) initiative and the Civic Nexus project the importance of developing an agreed plan with stakeholders. The plan should document how ICT will be rolled out across the LVO and be integrated with the strategic plan of the organisation, if one exists. If no strategic plan exists, which the research found to be the case for the majority of LVOs, one should be created.

\section{c. Participatory Action}

The focus of ICT initiatives should be on enabling and empowering members to do something that they were previously unable to do, or providing them with efficiencies with their current workload. Inclusion of members' views is paramount for the success of technological initiatives; they need to be able to contribute their expectations and feelings about new technical developments. Participatory action decrees that the needs of end-users should be considered at all stages of a CI initiative to ensure their adoption and use of ICT services. Many CI practitioners like Kretzmann and Mc Knight (1993), Gurstein (2008) and Stillman and French (2009) believe that stakeholder participation is an important aspect of application development. 


\section{d. Member Education and Training}

Education and training about the potential uses of ICT is vital for LVO members, if they are to participate in the development of technical solutions and subsequently adopt and use it successfully. Education and training programmes has a pivotal role to play in facilitating LVOs to identify ways to use technology, especially in organisations where members are apprehensive about using it. Many informants believe that until the membership of their LVO is ICT literate, technical applications will not be adopted in any significant manner. Scholars like Bourdieu (1996) and Smith (2007) expressed concerns about how the lack of education and training opportunities was stymieing the development of social and cultural capital in LVOs. Indeed, O'Donnell (2007) and the Wheel (2010) called for the development of education and training programmes for members of LVOs, as a mechanism to assist the diffusion of technology.

\section{e. ICT Development}

Many informants expressed views that a phased and iterative approach to ICT deployment was the most suitable for Irish LVOs as it allowed them to move forward slowly, yet with a definitive goal. This cautious, yet inclusive approach to ICT development was supported in the CI literature by Burt and Taylor (2000), Denison (2006) and Gurstein (2007). These scholars suggested that in order to ensure that these newly technically-enabled organisations retained their culture and embedded values, it was imperative that an evolutionary approach was adopted to ensure the transformational effects of ICT were embraced and made persistent at all levels of the organisation.

\section{f. Basic ICT Applications}

This point advocates how information is captured by the organisation and disseminated to its members through basic technologies. The findings from the data analysis show that capturing appropriate information within LVOs is difficult and becomes an even more challenging activity to sustain. Information capture and storage is a basic technical requirement for LVOs; however, the majority are reluctant to embrace this technological necessity. LVOs need to begin using database technologies to ensure that important organisational data is captured, stored and available for use, especially as the organisation evolves and grows. LVOs need to urgently capture important pieces of information that up to now have largely been in people's minds, and safely store them in a database for access and dissemination at a later stage. This form of knowledge management would assist LVOs to capture expertise and knowledge for diffusion between their members.

\section{g. Sophisticated Technologies}

Once LVOs have mastered the use of basic forms of ICT, they may need to evolve towards a more sophisticated level of technology use. These more complex technological developments usually involve the deployment of Web 2.0 and its associated technologies to provide services in areas like social capital development, economic opportunity creation and cultural retention, all of which have been identified by informants as important concerns for Irish LVOs. These sophisticated information systems usually involve the development of web enabled services that are accessible through the LVO's website or a hand held device. 
The development of social capital can be achieved through the creation of chat rooms, message boards, Facebook pages or Twitter. All of these technologies promote member interaction, collaboration and co-operation. Economic opportunities for raising finance are also available through mobilisation of appropriate forms of ICT; examples are on-line payment of membership, merchandise sales and retail of local lottery tickets. Some organisations have been using ICT more creatively to raise money. For example a LVO ran an event where the lowest unique bid over one thousand euro got to purchase that house for that price, after they had purchased a twenty euro ticket online.

\section{The ENCITE Model}

Figure 1.1 depicts the ENCITE model. It illustrates ENCITE's four lenses: Environment, Capital, ICT and End user, and shows the concepts and activities that are viewable through each one. These lenses were identified from grouping the acquired data into their respective areas and explicitly mentioned by informants as specific views of the respective LVOs which they considered useful for management, growth and development.

\section{Concepts/Activities}
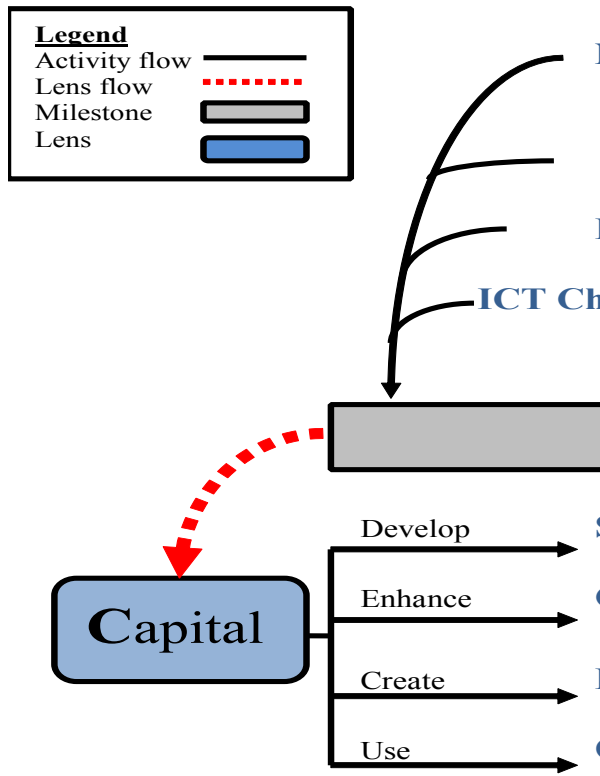

Social interaction

Cultural heritage

Economic opportunities

Other forms of capital

Education \& Training

Initial Project Plan

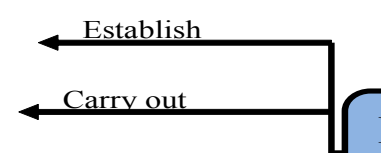

ENvironment

Identify

Select
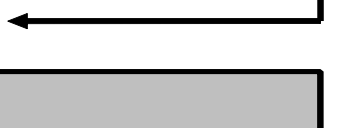


\section{Description of the ENCITE Model}

Figure 1.1 illustrates the proposed ENCITE model, which shows the interaction of the four lenses and their associated concepts and activities. Each lens provides LVOs with a unique way to look at their use of ICT and contains concepts and activities that are visible during their technical journey and useful for its completion. The concepts and activities in each lens interact together to enable LVOs to perform tasks that allow them to make progress on technical developments in an iterative and inclusive manner.

\section{Environment lens}

The first lens of the ENCITE model describes the LVO's environment. The Environment lens provides an operational view of the LVO, where stakeholders outline how their organisation operates, and conducts a SWOT analysis to establish an in-depth understanding of it. The outcome from the SWOT analysis is a starting point for the CI initiative. The Environment lens also examines the training and education needs of members and recognises that the success of LVO initiatives is dependent on having a technically literate membership. Often, this evaluation process leads to the identification of a CI champion, who is a technically capable member willing to lead ICT initiatives with support from other members of the organisation. Finally, this lens culminates in the development of an opportunities and project plan. The plan should specify the activities that the LVO needs to complete to address their technical requirements and provide a starting vision for the capital lens.

\section{Capital lens}

The second lens identifies the capital requirements of the organisation. The capital lens focuses on specifying the social, cultural and economic requirements of the LVO. This lens encourages LVOs to identify and evaluate opportunities that improve the social capital among its members, preserves and utilises their cultural heritage and ideals and facilitates the development of economic capital opportunities. These activities are the value added services that will improve and enhance the operation of the organisation and further empower their members. Organisations may also evaluate the use of socio-cultural constructionism through this lens, if education and training of members had been identified previously as a need of the LVO. The capital lens usually builds on the work carried out during the operational lens, although it is not a prerequisite. The capital lens assumes that the LVO has already identified and attained their basic operational needs, understands the potential uses of ICT and has started to identify tasks to be supported with technology, all of which have been specified in the opportunities and project plan. This lens culminates in the development of an analysis and design document.

\section{ICT lens}

The third lens of the model specifies the ICT focus of the model, which addresses how technology can facilitate the delivery of information and services to fulfil the needs and expectations of the LVO, as specified in the analysis and design document. The ICT lens encourages LVOs to evaluate appropriate technologies that may be used by them to support their function and empower their members. Initially, the ICT lens encourages applications to 
be developed that are easily adopted by members and actively diffused by them. The services that offer a quick return on investment and are most useful should be developed first, before more sophisticated applications are introduced. The ICT lens also ensures that consideration is given to appropriate principles of human-computer interaction; these should focus on ease of use and usability, especially where LVOs contain a relatively technically unsophisticated membership. The provision of a checklist of required system functionality and the subsequent inclusion of the end user's needs and expectations into the application will assist the development of a functionally complete and useful system.

\section{End user lens}

The fourth lens of the model outlines the end user or members' view of the model. The end user lens advocates a participatory approach to ICT development by LVOs, which includes members in all stages of application development. The end user lens evaluates the characteristics and individual nuances of LVO members and uses these findings to develop appropriate ICT initiatives, which they should find easy to adopt and use and, perhaps more importantly, sustain. To help LVO members adopt ICT applications, the end user lens ensures a focus is placed on the adoption of appropriate HCI principles to build on the work carried out in the ICT lens and to ensure the development of better and easier to use systems. The end user lens supports the continued evaluation of ICT developments by LVO members; it acknowledges that their contributions and feedback are important for the success of the initiative.

\section{Conclusion}

The ENCITE model provides researchers and academics with another way to think about the systematic use of ICT across communities and voluntary organisations. As the ENCITE model provides a holistic approach to technology adoption and diffusion, it will no doubt stimulate debate and provoke further interest in the development of supports that facilitate communities and LVOs to take responsibility for their own technological future. The ENCITE model will further the academic debate on the ways ICT can support voluntary organisations operation, assist with the development of services and empowering its members. At a practical level, the ENCITE model has been adopted and used as a technical template by a national voluntary organisation in Ireland; the initial feedback has been very positive in particular the technical education of its members, the retention of its historical culture and dissemination of best practices. The ENCITE model will facilitate voluntary organisations address what is clearly a complex socio-technical problem, identify opportunities for future technical developments and ensure these valuable organisations continue to make a meaningful contribution to Irish society.

\section{References}

Ahern, B. (2000) White Paper on a Framework for Supporting Voluntary Activity. In Taoiseach, A. (Ed.), Irish Government.

Asc, I. (2007) Access, Skills \& Content Initiative Guidelines \& Criteria. In Taoiseach, A. (Ed.), Irish Government. 
Anderson, E. (2000) Beyond homo economicus. New developments in theories of social norms: Philosophy and Public Affairs. 29(2), 170- 200

Bourdieu, P. (1983) Forms of capital in J. C. Richards (ed.) Handbook of Theory and Paper for the Sociology of Education. Greenwood Press, New York.

Burt, E. and Taylor, J. (2000) Information and Communication Technologies: Reshaping Voluntary Organizations? Non-profit Management \& Leadership. 11 (2), 131-143

Carroll, J.M. and Rosson, M.B. (1996) Developing the Blacksburg electronic village, Communications of the ACM, 39 (12), 69-74.

Carroll, J.M. and Rosson, M.B. (2007) Participatory Design in Community Informatics", The Journal of Community Informatics Special Issue on Participatory Design, pp. 243-261.

CUITA (2010) Community on the uptake of Information Technology In Agriculture and Rural Communities, Department of Agriculture, Fisheries and Food. Dublin

Denison, T. (2006) The Diffusion and Sustainability of Technology within Community Sector Organisations. International Journal of Knowledge, Culture and Change Management, 6 (3), 19-28.

Dochas, W. (2006) The case for better Community and Voluntary sector governance, White paper. The Wheel. Dublin.

E-Inclusion. (2005) e-Inclusion revisited: The local dimension of the Information Society. In Communities. Brussels.

Gilligan, R. (2005) Questioning the 'Rural' Adoption and Use of ICTs, in L. Haddon, B. Sapio, E. Mante-Meijer, L. Fortunati, and A. Kant, (eds.) Innovatory Users and Information and Communication Technologies. Springer-Verlag (London) Ltd.

Gurstein, M. (2000) Community Informatics: Enabling Communities with Information and Communications Technologies, Idea Group Publishing, Hershey PA.

Gurstein, M. (2007) What is Community Informatics (and Why Does It Matter)?. POLIMETRICA.

Irl, T. (2010) PreBudget submission, Irish Rural Link, http://www.socialjustice.ie/sites/ default/files/file/Budget/Budget\%202011/Pre-Budget\%20Submissions/Irish\%20Rural \%20Link\%20Pre\%20Budget\%20Submission\%202011.pdf.

Kretzmann, J. P., McKnight, J. L. (1993) Building Communities from the Inside Out: A Path towards Finding and Mobilizing a Community's Assets. Chicago, IL: ACTA Publications.

Mc Donald, T. J. (2012). The ENCITE model: Facilitating Irish Voluntary Organisations use ICT. Conference Proceedings- CIRN Community Informatics Conference "To measure or not to measure: that is the question": 9-11 November 2011, Monash Centre Prato, Italy.

Morse, S. (2004) Smart Communities: How Citizens and Local Leaders Can Use Strategic Thinking to Build a Brighter Future. Jossey Bass, Wiley 
O'Donnell, S. (2000) Small media, big network: Alternative media and social movements on the Internet. Unpublished PhD thesis. Dublin City University, Dublin.

O'Donnell, S. (2001) Towards an Inclusive Information Society in Europe: The Role of Voluntary Organisations. Dublin: Itech Paper.

O'Donnell, S. and Trench, B. (1999) Voluntary and community organisations in Ireland's Information Society. Coleraine: University of Ulster, Association for Voluntary Action Paper in Ireland.

Stillman, L. and French, R. (2007) Doing IT Better: How does the welfare sector act with technology? Community informatics - prospects for communities and action, 4th CIRN Community Informatics Conference. Monash Prato Centre, 5-7 November 2007, Prato, Italy.

Smith, M. K. (2007) Social capital. The Encyclopaedia of Informal Education.

Toyama, K. (2009) What constitutes good ICTD paper? Information Technologies and International Development, 5(3):82-94. http://itidjournal.org/itid/article/view/ $382 / 178$.

Wheel, T. (2010) ICTpoint - IT Knowhow for the Non-Profit and Charity Sector. http:// www.wheel.ie/you-and-your-organisation/technology. 